Canadian

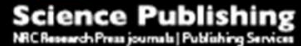

Canadian Journal of Physiology and Pharmacology Revue canadienne de physiologie et pharmacologie

\title{
Nerve growth factor enhances the CRE-dependent transcriptional activity activated by nobiletin in PC-12 cells
}

\begin{tabular}{|r|l|}
\hline Journal: & Canadian Journal of Physiology and Pharmacology \\
\hline Manuscript ID & cjpp-2015-0394.R1 \\
\hline Danuscript Type: & Article \\
\hline Complete List of Authors: & $\begin{array}{l}\text { Takito, Jiro; Showa University, Oral Anatomy and Developmental Biology, } \\
\text { School of Dentistry } \\
\text { Kimura, Junko; School of Pharmaceutical Sciences, University of Shizuoka, } \\
\text { Department of Medical Biochemistry } \\
\text { Kajima, Koji; Sankyo Holdings Co.,Ltd, } \\
\text { Uozumi, Nobuyuki; Tohoku University, Department of Biomolecular } \\
\text { Engineering, Graduate School of Engineering } \\
\text { Watanabe, Makoto; Kansei Fukushi Research Center, Tohoku Fukushi } \\
\text { University } \\
\text { Yokosuka, Akihito; School of Pharmacy, Tokyo University of Pharmacy and } \\
\text { Life Sciences, Laboratory of Medicinal Plant Science } \\
\text { Mimaki, Yoshihiro; School of Pharmacy, Tokyo University of Pharmacy and } \\
\text { Life Sciences, Laboratory of Medicinal Plant Science } \\
\text { Nakamura, Masanori; Showa University, Department of Oral Anatomy and } \\
\text { Developmental Biology, School of Dentistry } \\
\text { Ohizumi, Yasushi; Kansei Fukushi Research Center, Tohoku Fukushi } \\
\text { University }\end{array}$ \\
\hline Keyword: & $\begin{array}{l}\text { alzheimer disease, CRE-dependent transcription, nerve growth factor, } \\
\text { nobiletin, PC12 cells }\end{array}$ \\
\hline
\end{tabular}


Nerve growth factor enhances the CRE-dependent transcriptional activity

activated by nobiletin in PC-12 cells

Jiro Takito, Junko Kimura, Koji Kajima, Nobuyuki Uozumi, Makoto Watanabe,

Akihito Yokosuka, Yoshihiro Mimaki, Masanori Nakamura, and Yasushi Ohizumi

\section{Affiliation}

J. Takito ${ }^{1}$ and M. Nakamura. Department of Oral Anatomy and Developmental

Biology, School of Dentistry, Showa University, 1-5-8 Hatanodai, Shinagawa, Tokyo,

Japan

J. Kimura and Y. Ohizumi. Department of Medical Biochemistry, School of

Pharmaceutical Sciences, University of Shizuoka, 52-1 Yada, Suruga-ku, Shizuoka,

Japan

K. Kajima. Sankyo Holdings Co.,Ltd, 573-13 Denbou, Fuji-shi, Shizuoka, Japan

N. Uozumi. Department of Biomolecular Engineering, Graduate School of 
Engineering, Tohoku University, 6-6-07 Aobayama, Sendai, Miyagi, Japan

M. Watanabe and Y. Ohizumi. Kansei Fukushi Research Center, Tohoku Fukushi

University, 6-149-1 Kunimigaoka, Aoba-ku, Sendai, Miyagi, Japan

A. Yokosuka and Y. Mimaki. Laboratory of Medicinal Plant Science, School of

Pharmacy, Tokyo University of Pharmacy and Life Science, 1432-1 Horinouchi,

Hachioji, Tokyo, Japan.

\section{${ }^{1}$ Corresponding author}

Jiro Takito, Ph.D.

Department of Oral Anatomy and Developmental Biology, School of Dentistry, Showa

University, 1-5-8 Hatanodai, Shinagawa, Tokyo, 142-8555 Japan

E-mail: takito@dent.showa-u.ac.jp Phone: +8137848153 Fax: +81 37858190 


\section{Abstract:}

Prevention and treatment of Alzheimer disease are urgent problems for elderly people in developed countries. We previously reported that nobiletin, a poly-methoxylated flavone from the citrus peel, improved the symptoms in various types of animal models of memory loss and activated the cAMP responsive element (CRE)-dependent transcription in PC12 cells. Nobiletin activated the cAMP/PKA/MEK/Erk/MAPK signaling pathway without using the TrkA signaling activated by nerve growth factor (NGF). Here we examined the effect of combination of nobiletin and NGF on the CRE-dependent transcription in PC12 cells. Although NGF alone had little effect on the CRE-dependent transcription, NGF markedly enhanced the CRE-dependent transcription induced by nobiletin. The NGF-induced enhancement was neutralized by a TrkA antagonist, K252a. This effect of NGF was effective on the early signaling event elicited by nobiletin. These results suggested that there was crosstalk between NGF and nobiletin signaling in activating the CRE-dependent transcription in PC12 cells. 
Key words:

alzheimer disease, CRE-dependent transcription, flavone, nerve growth factor, neuron,

nobiletin, PC12 cells. 


\section{Introduction}

Alzheimer disease (AD) is a neurodegenerative disorder characterized by the impaired cognition and memory loss. The progression of AD takes more than 10 years and the illness results in a devastating death of the patient (Huang and Mucke 2012).

Because there are no effective treatments to prevent, halt, or cure the $\mathrm{AD}$, diversified

efforts for researches and drug developments are required. Learning and memory depend on the establishment of long-term potentiation in the hippocampus (Kandel 2001). Biochemically, long-term potentiation is established by the initiation of the cyclic AMP (cAMP)-dependent signal transduction and the subsequent transcription of target genes via the cAMP-response element (CRE)-binding protein. CREB-mediated transcription is involved not only in learning and memory, but also in survival, differentiation, dendritic outgrowth and synaptic plasticity(Shaywitz and Greenberg 1999). Thus, it is reasonable to assume that the substance that stimulates the CRE-dependent transcription in vitro becomes a potential curative medicine for treating neurodegenerative disorders. In line with this idea, we screened natural resources and 
found that nobiletin, a citrus flavonoid, activated the CRE-dependent transcription in PC12D cells, a rat pheochromocytoma cell line (Nagase et al. 2005a). As expected, nobiletin has been proved to improve the memory deterioration in beta-amyloid infused AD model rat (Matsuzaki et al. 2006), bulbectomy-induced cholinergic neurodegenerative mice (Nakajima et al. 2007), transgenic mouse model of AD (Onozuka et al. 2008), and Parkinson disease model mice (Yabuki et al. 2014). Importantly, administration of nobiletin-rich Chinpi prevented a progression of the cognitive impairment of donepezil-treated AD patients (Seki et al. 2013). Thus, nobiletin is a good candidate for anti-dementia drug (Nakajima et al. 2014; Ohizumi 2015; Yamakuni et al. 2008).

Nerve growth factor (NGF) is a neurotrophin that promotes survival, neurite outgrowth, and synthesis of neurotransmitters in neurons. These actions of NGF are mediated by binding to the high affinity receptor, the tropomyosin-regulating kinase A (TrkA)(Chao 2003; Huang and Reichardt 2003). Chronic deprivation of NGF in mice exhibits the sympotoms of AD such as neuronal loss, tau hyperphosphorylation, and deposition of $\beta$-amyloid plaques (Capsoni et al. 2002; Ruberti et al. 2000). Hence, NGF 
is one of candidates to combat against AD (Rossner et al. 1998). But, previous studies reported that NGF had no effect on the activation of CRE-dependent transcription in PC12 cells after short-term treatment (Chang et al. 2003; Minneman et al. 2000). Our screen accidentally identified NGF that potentiated the activity of nobiletin in vitro.

Here we report that NGF enhances the nobiletin-induced CRE-dependent transcription in PC12 cells. This raises the hypothesis that the in vivo effect of nobiletin to improve the memory deficit is partly mediated by the endogenous NGF. 


\section{Materials and Methods}

\section{Materials}

Nobiletin was extracted and isolated as described previously (Nagase et al. 2005a). Anti-ERK1/2 antibody was from Promega (Madison, WI) and Phospho-p44/42 MAPK (T202/Y204) antibody was from Cell Signaling Technology (Danvers, MA). PD 98059 was from Cayman Chemical (Ann Arbor, MI). Mouse NGF 7S and K252a were obtained from Alomone Labs (Jerusalem, Israel). PC12 cell was obtained from the Riken BioResource center (Tsukuba, Ibaraki, Japan).

\section{Measurements of CRE-dependent transcriptional activity in PC12 cells}

To measure the CRE-dependent transcriptional activity in PC12 cells, we adopted the reporter gene assay using the pCRE, firefly luciferase reporter plasmid (Clontech Laboratories Inc. Mountain View, CA) as described previously (Fujiwara et al. 2011). In short, PC12 cells were maintained in Advanced-DMEM (Gibco by Life Technologies,

Grand Island, NY), 10\% fetal bovine serum, 5\% horse serum and 1\% Penicillin-Streptomycin-Glutamine (Gibco) in a $5 \% \mathrm{CO}_{2}$ incubator at $37^{\circ} \mathrm{C}$. The cells were seeded in 96-well plates at a density of $2 \times 10^{4}$ cells per well. Next day, PC12 cells 
were transfected with $0.2 \mathrm{mg} \mathrm{pCRE}$ and $0.04 \mathrm{mg}$ phRG-TK (a renilla luciferase, Promega, Madison, WI) per well using LipofectAMINE 2000 according to the manufacturer's protocol (Invitrogen, Carlsbad, CA). Five hours after the transfection, the culture medium was changed into a fresh medium containing Advanced-DMEM, $1 \%$ fetal bovine serum, $1 \%$ horse serum. A day after the transfection, the cells were stimulated with nobiletin or NGF in Advanced-DMEM, 1\% fetal bovine serum, $1 \%$ horse serum. An equal volume of dimethyl sulfoxide was added for the control. For the inhibitor experiments, the cells were preincubated with an inhibitor for $30 \mathrm{~min}$ before the addition of nobiletin or NGF. Five hours after the addition of nobiletin, the cell lysates were recovered and used for the measurements of luciferase activities.

Luminescence was determined using Dual-Luciferase Reporter Assay System according to the manufacturer's protocol (Promega, Madison, WI) in Appliskan (Thermo Electric Corp.). The firefly luciferase activities were normalized to those of Renilla luciferase activities and were presented as relative values against the control. The measurements were performed in quadruplicate. The results presented are representative of at least three times of independent assays. 


\section{Western blot}

PC12 cells were seeded at $3 \times 10^{5}$ cells in $3 \mathrm{ml}$ of Advanced-DMEM, 10\% fetal

bovine serum, $5 \%$ horse serum and $1 \%$ Penicillin-Streptomycin-Glutamine in a 6 -well

plate. Next day, the culture medium was changed into a fresh medium containing

Advanced-DMEM, 1\% fetal bovine serum, 1\% horse serum. A day after the medium

change, the cells were stimulated with various test substances in Advanced-DMEM, 1\%

fetal bovine serum, $1 \%$ horse serum. For the inhibitor experiments, the cells were

preincubated with an inhibitor for $30 \mathrm{~min}$ before the addition of nobiletin or NGF. The

cells were washed once with PBS and recovered by scraping with $0.1 \mathrm{ml}$ of $1 \% \mathrm{NP}-40$,

$5 \mathrm{mM} \mathrm{MgCl}_{2}, 150 \mathrm{mM} \mathrm{NaCl}, 5 \%$ glycerol, $0.32 \mu \mathrm{M}$ okadaic acid, $0.1 \mu \mathrm{M}$ calyculinA,

$0.8 \mathrm{mM}$ NaF, $0.25 \mathrm{mM}$ sodium orthovanadate and $25 \mathrm{mM}$ Tris- $\mathrm{HCl}(\mathrm{pH}$ 7.2) with

protease inhibitor cocktails (Sigma-Aldrich, St. Louis, MO) at the indicated times. The

recovered cells were vortexed for $20 \mathrm{sec}$ and incubated on ice for $5 \mathrm{~min}$. The cells were

centrifuged at $16,000 \mathrm{~g}$ for $15 \mathrm{~min}$ at $4^{\circ} \mathrm{C}$, and the resulting supernatant was used for

western blot. A SuperSep ${ }^{\mathrm{TM}}$ Ace $12.5 \%$ gel (Wako) was used for protein separation by

SDS-PAGE, and separated proteins were transferred to Immobilon-FL (Millipore 
Corporation, Billerica, MA). The PVDF membrane was blocked with 3\% bovine serum albumin (Sigma-Aldrich) followed by incubation with anti-phospho-p44/42 MAPK (T202/Y204) antibody and secondary antibodies. Proteins were detected by chemiluminescence using the $\mathrm{ECL}^{\mathrm{TM}}$ prime western blotting detection reagent (GE Healthcare, Buckinghamshire, UK) on a ChemiDoc XRS (Bio-Rad Laboratories, Hercules, CA). After detecting the phospho-Erk signal, the membrane was stripped off according to the protocol provided by the manufacturer and reprobed with the anti-ERK1/2 antibody. The obtained digital images were processed using Photoshop CS (Adobe, CA).

\section{Statistical analysis}

The data are expressed as the means $\pm \mathrm{SD}$. Comparison between the groups were performed using paired Student's t-test on Microsoft Excel (Microsoft, Redmond, WA) and the ANOVA (Anova4 on the Web; http://www.hju.ac.jp/ kiriki/anova4). 


\section{Results}

\section{NGF enhanced the CRE-dependent transcription induced by nobiletin}

The effect of NGF was examined on the CRE-dependent transcription induced by nobiletin in PC12 cells (Fig.1A). As reported previously (Nagase et al. 2005a), nobiletin at $30 \mu \mathrm{M}$ induced an increase by over 20 -fold in the CRE-dependent transcription.

Combination of NGF at 30 and $100 \mathrm{nM}$ with nobiletin significantly enhanced the activity induced by nobiletin. In the absence of nobiletin, NGF from 1 to $100 \mathrm{nM}$ had no effect on the activity.

The effect of nobiletin on the CRE-dependent transcription in PC12 cells was usually tested at 5 hours after the addition of nobiletin. To confirm the effect of NGF on the nobiletin-induced transcriptional activity, we examined the effect of nobiletin at various time points. As shown in Fig. 1B, NGF at $50 \mathrm{nM}$ significantly enhanced the CRE-dependent transcription induced by nobiletin from 1 to 5 hours after the addition.

NGF itself showed no effect on the activity during the time examined. Nobiletin lost its activating effect of the CRE-dependent transcription at 48 hours after the addition. The 
activating effect of NGF also disappeared at 48 hours after the addition. In contrast,

NGF slightly increased the CRE-dependent transcription at 48 hours after the addition.

This coincided with the result that NGF increased the transcription after long-term

treatment in PC12 cells (Chang et al. 2006).

\section{Effect of K252a and PD98059 on the NGF-induced enhancement of}

\section{CRE-dependent transcription}

We next examined the effect of K252a, a TrkA antagonist, on the nobiletin-induced

activation of the CRE-dependent transcription in the presence and absence of NGF (Fig.

2). K252a showed no effect on the nobiletin-induced activation of the CRE-dependent

transcription in the absence of NGF. On the other hand, in the presence of NGF, K252a

dose-dependently inhibited the enhancement of nobiletin-induced activity by NGF to

the basal level. The result suggests that the effect of NGF on the nobiletin-induced

activation is mediated by TrkA receptor and that nobiletin signaling crosstalks with

NGF signaling downstream of TrkA.

Nobiletin signaling for the activation of CRE-dependent transcription involves Erk 
signaling (Nagase et al. 2005b). So, we examined the effect of TrkA antagonist and Erk antagonist on the nobiletin-induced activation in the presence and absence of NGF (Fig.

3A and 3B). In the absence of NGF, an Erk inhibitor, PD98059 dose-dependently inhibited the nobiletin-induced activation of the CRE-dependent transcription as expected. Coincided with the results in Fig. 2, K252a had little effect on the nobiletin-induced activation. Interestingly, there was no difference in the inhibitory effect of PD98059 with and without K252a (Fig. 3A). In the presence of NGF, K252a cancelled the NGF-induced enhancement of CRE-dependent transcription activity (Fig. 3B). As expected, PD98059 dose-dependently inhibited the nobiletin-induced CRE-dependent transcription activity. K252a combined with PD98085 showed stronger inhibitory effect than that of PD98085 alone (Fig. 3B). The results accord with a notion that TrkA A signaling crosstalks with nobiletin signaling upstream of Erk signaling.

\section{Effect of NGF and nobiletin on Erk phosphorylation}

The effect of NGF and nobiletin was examined on Erk phosphorylation in PC12

cells by Western blot. As we reported previously (Nagase et al. 2005b), nobiletin 
moderately increased Erk phosphorylation at 15, 30, and 60 min after the addition (Fig.

4A). NGF strongly increased Erk phosphorylation at all time points examined. Erk

phosphorylation stimulated by nobiletin combined with NGF was higher than that

stimulated by NGF or nobiletin alone at 30 and $60 \mathrm{~min}$ after the addition. The results

suggest that nobiletin and NGF cooperatively upregulate the Erk phosphorylation.

To characterize the cooperativity of nobiletin and NGF in activating Erk, we tested

the effect of various inhibitors on the nobiletin-induced phosphorylation of Erk (Fig.

4B). Erk phosphorylation stimulated by nobiletin was inhibited by PD98059 and

PD98059 combined with K252a, but not K252a. In contrast, Erk phosphorylation

stimulated by nobiletin combined with NGF was inhibited by both K252a and PD98059.

Interestingly, the inhibitory effect of K252a combined with PD98059 was stronger than

that of K252a or PD98059 alone. These inhibitory patterns of Erk phosphorylation by

PD98059 and K252a were similar to those obtained by measuring the CRE-dependent

transcription (Fig. 3A and 3B).

NGF interacts with the early signaling event elicited by nobiletin 
All results described above were obtained by adding nobiletin and NGF at the same time. In this experiment, PC12 cells were first stimulated by nobiletin, and then the cells were received the following addition of NGF at various time points (Fig. 5). The delayed addition of NGF significantly enhanced the nobiletin-induced CRE-dependent transcription within $60 \mathrm{~min}$ of nobiletin addition. But NGF added after $60 \mathrm{~min}$ of nobiletin addition showed no activating effect on the nobiletin-induced CRE-dependent transcription. The results suggest that the NGF signal could interact only with the early event(s) of nobiletin signal in PC12 cells.

\section{Effect of NGF preincubation on the nobiletin-induced CRE-dependent}

transcription

To examine the effect of NGF preincubation on the nobiletin-induced

CRE-dependent transcription, PC12 cells were preincubated with NGF for 10 to 120

min. Then, the CRE-dependent transcription was started by changing the culture

medium with nobiletin alone (Fig. 6). The NGF preincubation significantly enhanced 
the CRE-dependent transcription induced by nobiletin at all time points examined. The result suggests that the signal elicited by NGF lasts at least for 2 hours for the crosstalk with nobiletin signaling.

Using the same preincubation protocol, we also measured the CRE-dependent transcription started by nobiletin plus NGF (Fig. 6). The coexistence of NGF with nobiletin enhanced the effect of NGF preincubated for 0,10 and $30 \mathrm{~min}$, but had no effect on the effect of NGF preincubated for 60 and $120 \mathrm{~min}$. These results may suggest the complexity of NGF action on the CRE-dependent transcription induced by nobiletin. 


\section{Discussion}

In this study, we showed for the first time that NGF enhanced the nobiletin-induced

CRE-dependent transcription in PC12 cells. The basis of NGF action on PC12 cells was explained by the presence of high affinity binding of ${ }^{125} \mathrm{I}-\mathrm{NGF}$ on the cell surface of PC12 cells (Zhou et al. 1995). NGF rapidly induced phosphorylation of TrkA through which NGF transmitted signals leading to differentiation and dendrite outgrowth. It was controversial whether NGF increased the CRE-dependent transcription or not. Previous study showed that NGF treatment for $4 \mathrm{~h}$ had little effect on the CRE-dependent transcription in PC12 cells stably transfected with CRE-luciferase plasmid (Minneman et al. 2000). On the other hand, Chang et al. reported that the treatment of NGF for $5 \mathrm{~h}$ increased the base level of CRE-dependent transcription by 2-fold in PC12 cells stably transfected with CRE-luciferase plasmid (Chang et al. 2003). But, this increase was insensitive to K252a. NGF exerted its activating effect (over 20-fold) on the CRE-dependent transcription after 48 h, which was cancelled by K252a. Thus, NGF appeared to have no effect on the CRE-dependent transcription in the undifferentiated PC12 cells, but it increased the CRE-dependent transcription in the differentiated PC12 
cells. By our hands, NGF treatment within $5 \mathrm{~h}$ had little effect on the CRE-dependent transcription (Fig. 1A and 1B). So, we conclude that short-term treatment of NGF has little effect on the CRE-dependent transcription in PC12 cells transiently transfected with CRE-luciferase plasmid.

The NGF signaling via TrkA induced the enhancement of nobiletin-induced CRE-dependent transcription, because K252a specifically inhibited the NGF-induced enhancement (Fig.2). One of the common signaling events of nobiletin and NGF was Erk phosphorylation. As expected, nobiletin and NGF cooperatively increased the Erk phosphorylation (Fig. 4A). The enhanced Erk phosphorylation was inhibited by K252a and PD98059 (Fig. 4B). This coincided well with the results of measuring the CRE-dependent transcription (Fig. 3B). Thus, the crosstalk of NGF and nobiletin signaling probably occurred between TrkA and Erk phosphorylation.

Although the target molecule of nobiletin was unknown, cAMP appeared to be a key molecule in the action of nobiletin. Because CREB phosphorylation depended on the intracellular cAMP (Hagiwara et al. 1992), dibutyryl cAMP caused rapid and transient CRE-dependent transcription in PC12 cells (Chang et al. 2006). Nobiletin significantly 
increased the intracellular cAMP in the presence of forskolin (Nagase et al. 2005b). On the other hand, nobiletin inhibited the phoshodiesterase activity in the bovine heart.

Accordingly, we previously concluded that nobiletin used the cAMP/PKA/MEK/Erk/MAPK/CREB pathway. Authentic NGF signaling mediated TrkA/Ras/Raf/MEK/Erk/MAPK/CREB pathway (Huang and Reichardt 2003). Thus, the signaling event between TrkA and ERK might be involved in the crosstalk of nobiletin with NGF. Activation of MAPK by cAMP depended on B-Raf and Rap-1 in PC12 cells (Peraldi et al. 1995; Stork and Schmitt 2002; Vossler et al. 1997). It might be useful to test whether nobiletin transactivates B-raf and Rap-1 in future.

This study showed that nobiletin crosstalked with NGF signaling in the undifferentiated PC12 cells. Our preliminary experiments indicated that NGF also enhanced the nobiletin-induced CRE-dependent transcription in the differentiated PC12 cells that were cultured with NGF for 3 days (unpublished data). Hence, NGF probably acted both the undifferentiated and differentiated PC12 cells in vitro. In vivo, nobiletin not only improved various types of model animals of memory deficits (Matsuzaki et al. 2006; Nakajima et al. 2007; Onozuka et al. 2008; Yabuki et al. 2014), but also exerted 
neuroprotective action against cerebral ischemia-reperfusion injury (Yasuda et al. 2014).

Nobiletin showed neurotrophic actions such as dendritic outgrowth (Nagase et al.

2005a) and the rescue of degenerated cholinergic neurons (Nakajima et al. 2007).

Crosstalk of nobiletin and NGF signaling in the CRE-dependent transcription might

provide an explanation for these extensive actions of nobiletin. The concentration of

nobiletin in serum and the brain at 3 min after intraperitoneal administration to mice

was 18 and $55 \mu \mathrm{M}$, respectively (Saigusa et al. 2011). Because the concentration of

nobiletin in the brain was similar to that one used in the present study $(10$ and $30 \mu \mathrm{M})$,

nobiletin in the brain might work with endogenous NGF. Nobiletin might strengthen

and/or complement the action of endogenous NGF. In this regard, it might be interesting

to identify the genes commonly regulated by nobiletin and NGF. Gene expression

analysis using microarray revealed that nobiletin upregulated Ddit3 and Trib3, while it

downregulated Txnip (Nemoto et al. 2013). All thee genes were upregulated in the

sympathetic neurons after NGF withdrawal that was associated with cell death

(Kristiansen et al. 2011; Mayumi-Matsuda et al. 1999). Nobiletin downregulated Txnip

and suppressed the tunicamycin-induced apoptosis in human neuroblastoma cells (Ikeda 
et al. 2013). Therefore, future studies on the regulation of gene transcription of these

genes might help to understand the nature of crosstalk between nobiletin and NGF.

In conclusion, we have shown for the first time that NGF signaling via TrkA

enhanced the nobiletin-induced CRE-dependent transcription in PC12 cells. The

mechanisms involved in crosstalk between NGF and nobiletin signaling require further

investigation.

\section{Acknowledgements}

We thank Ms. Michi Kawada for her excellent technical assistance.

\section{Conflict of Interest}

The authors declare no conflict of interest. 


\section{References}

Capsoni, S., Giannotta, S., and Cattaneo, A. 2002. Nerve growth factor and galantamine ameliorate early signs of neurodegeneration in anti-nerve growth factor mice.

Proc. Natl. Acad. Sci. U S A. 99(19): 12432-12437.

Chang, J.H., Mellon, E., Schanen, N. C., and Twiss, J. L. 2003. Persistent TrkA activity is necessary to maintain transcription in neuronally differentiated PC12 cells. J.

Biol. Chem. 278(44): 42877-42885.

Chang, J.H., Vuppalanchi, D., van Niekerk, E., Trepel, J. B., Schanen, N. C., and Twiss, J.L. 2006. PC12 cells regulate inducible cyclic AMP (cAMP) element repressor expression to differentially control cAMP response element-dependent transcription in response to nerve growth factor and cAMP. J. Neurochem. 99(6): $1517-1530$.

Chao, M.V. 2003. Neurotrophins and their receptors: a convergence point for many signalling pathways. Nat. Rev. Neurosci. 4(4): 299-309.

Fujiwara, H., Kogure, A., Sakamoto, M., Yamakuni, T., Mimaki, Y., Murata, K., et al. 
2011. Honeybee royal jelly and nobiletin stimulate CRE-mediated transcription in ERK-independent and -dependent fashions, respectively, in PC12D cells. J.

Pharmacol. Sci. 116(4): 384-387.

Hagiwara, M., Alberts, A., Brindle, P., Meinkoth, J., Feramisco, J., Deng, T.,et al.

Transcriptional attenuation following cAMP induction requires PP-1-mediated dephosphorylation of CREB. Cell, 70(1): 105-113.

Huang, E.J., and Reichardt, L. F. 2003. Trk receptors: roles in neuronal signal transduction. Annu. Rev. Biochem. 72:609-642.

Huang, Y., and Mucke, L. 2012. Alzheimer mechanisms and therapeutic strategies. Cell, 148(6): 1204-1222.

Ikeda, A., Nemoto, K., Yoshida, C., Miyata, S., Mori, J., Soejima, S., et al. 2013. Suppressive effect of nobiletin, a citrus polymethoxyflavonoid that downregulates thioredoxin-interacting protein expression, on tunicamycin-induced apoptosis in SK-N-SH human neuroblastoma cells. Neurosci. Lett. 549:135-139.

Kandel, E.R. 2001. The molecular biology of memory storage: a dialogue between genes and synapses. Science, 294(5544): 1030-1038. 
Kristiansen, M., Menghi, F., Hughes, R., Hubank, M., and Ham, J. 2011. Global analysis of gene expression in NGF-deprived sympathetic neurons identifies molecular pathways associated with cell death. BMC Genomics, 12:551.

Matsuzaki, K., Yamakuni, T., Hashimoto, M., Haque, A. M., Shido, O., Mimaki, Y., et al. 2006. Nobiletin restoring beta-amyloid-impaired CREB phosphorylation rescues memory deterioration in Alzheimer's disease model rats. Neurosci. Lett. 400(3): 230-234.

Mayumi-Matsuda, K., Kojima, S., Suzuki, H., and Sakata, T. 1999. Identification of a novel kinase-like gene induced during neuronal cell death. Biochem. Biophys.

Res. Commun. 258(2): 260-264.

Minneman, K. P., Lee, D., Zhong, H., Berts, A., Abbott, K. L., and Murphy, T. J. 2000. Transcriptional responses to growth factor and G protein-coupled receptors in PC12 cells: comparison of alpha(1)-adrenergic receptor subtypes. J. Neurochem. 74(6): 2392-2400.

Nagase, H., Omae, N., Omori, A., Nakagawasai, O., Tadano, T., Yokosuka, A., et al. 2005a. Nobiletin and its related flavonoids with CRE-dependent 
transcription-stimulating and neuritegenic activities. Biochem. Biophys. Res.

Commun. 337(4): 1330-1336.

Nagase, H., Yamakuni, T., Matsuzaki, K., Maruyama, Y., Kasahara, J., Hinohara, Y., et al. 2005b. Mechanism of neurotrophic action of nobiletin in PC12D cells.

Biochemistry, 44(42): 13683-13691.

Nakajima, A., Ohizumi, Y., and Yamada, K. 2014. Anti-dementia Activity of Nobiletin,

a Citrus Flavonoid: A Review of Animal Studies. Clin. Psychopharmacol.

Neurosci. 12(2): 75-82.

Nakajima, A., Yamakuni, T., Haraguchi, M., Omae, N., Song, S. Y., Kato, C., et al.

Nobiletin, a citrus flavonoid that improves memory impairment, rescues

bulbectomy-induced cholinergic neurodegeneration in mice. J. Pharmacol. Sci.

105(1): 122-126.

Nemoto, K., Ikeda, A., Yoshida, C., Kimura, J., Mori, J., Fujiwara, H., et al. 2013.

Characteristics of nobiletin-mediated alteration of gene expression in cultured cell

lines. Biochem. Biophys. Res. Commun. 431(3): 530-534.

Ohizumi, Y. 2015. [A new strategy for preventive and functional therapeutic methods 
for dementia--approach using natural products] in Japanese. Yakugaku Zasshi,

135(3): 449-464.

Onozuka, H., Nakajima, A., Matsuzaki, K., Shin, R. W., Ogino, K., Saigusa, D., et al.

2008. Nobiletin, a citrus flavonoid, improves memory impairment and Abeta pathology in a transgenic mouse model of Alzheimer's disease. J. Pharmacol. Exp.

Ther. 326(3): 739-744.

Peraldi, P., Frodin, M., Barnier, J. V., Calleja, V., Scimeca, J. C., Filloux, C., et al. 1995.

Regulation of the MAP kinase cascade in PC12 cells: B-Raf activates MEK-1

(MAP kinase or ERK kinase) and is inhibited by cAMP. FEBS Lett. 357(3):

290-296.

Rossner, S., Ueberham, U., Schliebs, R., Perez-Polo, J. R., and Bigl, V. 1998. The regulation of amyloid precursor protein metabolism by cholinergic mechanisms and neurotrophin receptor signaling. Prog. Neurobiol. 56(5): 541-569.

Ruberti, F., Capsoni, S., Comparini, A., Di Daniel, E., Franzot, J., Gonfloni, S., et al. 2000. Phenotypic knockout of nerve growth factor in adult transgenic mice reveals severe deficits in basal forebrain cholinergic neurons, cell death in the 
spleen, and skeletal muscle dystrophy. J. Neurosci. 20(7): 2589-2601.

Saigusa, D., Shibuya, M., Jinno, D., Yamakoshi, H., Iwabuchi, Y., Yokosuka, A., et al. 2011. High-performance liquid chromatography with photodiode array detection for determination of nobiletin content in the brain and serum of mice administrated the natural compound. Anal. Bioanal. Chem. 400(10): 3635-3641.

Seki, T., Kamiya, T., Furukawa, K., Azumi, M., Ishizuka, S., Takayama, S., et al. 2013. Nobiletin-rich Citrus reticulata peels, a kampo medicine for Alzheimer's disease: a case series. Geriatr. Gerontol. Int. 13(1): 236-238.

Shaywitz, A.J., and Greenberg, M.E. 1999. CREB: a stimulus-induced transcription factor activated by a diverse array of extracellular signals. Annu. Rev. Biochem. 68:821-861.

Stork, P.J., and Schmitt, J.M. 2002. Crosstalk between cAMP and MAP kinase signaling in the regulation of cell proliferation. Trends Cell. Biol. 12(6): 258-266.

Vossler, M.R., Yao, H., York, R.D., Pan, M.G., Rim, C.S., and Stork, P.J. 1997. cAMP activates MAP kinase and Elk-1 through a B-Raf- and Rap1-dependent pathway. Cell, 89(1): 73-82. 
Yabuki, Y., Ohizumi, Y., Yokosuka, A., Mimaki, Y., and Fukunaga, K. 2014. Nobiletin treatment improves motor and cognitive deficits seen in MPTP-induced Parkinson model mice. Neuroscience, 259:126-141.

Yamakuni, T., Nakajima, A., and Ohizumi, Y. 2008. Pharmacological action of nobiletin, a component of AURANTII NOBILIS PERICARPIUM with anti-dementia activity, and its application for development of functional foods. Nihon Yakurigaku Zasshi, 132(3): 155-159.

Yasuda, N., Ishii, T., Oyama, D., Fukuta, T., Agato, Y., Sato, A., et al. 2014. Neuroprotective effect of nobiletin on cerebral ischemia-reperfusion injury in transient middle cerebral artery-occluded rats. Brain Res. 1559:46-54.

Zhou, J., Valletta, J.S., Grimes, M.L., and Mobley, W.C. 1995. Multiple levels for regulation of TrkA in PC12 cells by nerve growth factor. J. Neurochem. 65(3): $1146-1156$. 


\section{Legends to Figures}

\section{Fig. 1}

NGF enhanced the nobiletin-induced CRE-dependent transcription in PC12 cells. (A)

Dose response of NGF on the CRE-dependent transcription induced by nobiletin. PC12

cells were transiently transfected with a CRE reporter plasmid and incubated with the

indicated concentration of NGF in the presence of dimethyl sulfoxide (O) or $30 \mu \mathrm{M}$

nobiletin $(\bigcirc)$ for $5 \mathrm{~h}$. *Significantly different from the value without NGF, $p<0.05$.

(B) Time-course of the CRE-dependent transcription induced by nobiletin and NGF.

PC12 cells transiently transfected with a CRE reporter plasmid were incubated for the

indicated times with dimethyl sulfoxide ( $\mathbf{\square}), 50 \mathrm{nM}$ NGF $(\square), 10 \mu \mathrm{M}$ nobiletin $(\boldsymbol{O})$, or

$50 \mathrm{nM}$ NGF plus $10 \mu \mathrm{M}$ nobiletin $(\bigcirc)$. The luciferase activity was relative to the

control value. The assay was performed in quadruplicate. The value is expressed as an

average. The bar represents S.D. Significant differences among the four groups were

evaluated using ANOVA. *Significantly different from the value with the other three

groups, $p<0.01$. 


\section{Fig. 2}

The effect of K252a on the CRE-dependent transcription induced by nobiletin.

PC12 cells transiently transfected with a CRE reporter plasmid were preincubated with the indicated concentrations of K252a for $30 \mathrm{~min}$. Then, the medium was changed into fresh one that contained $10 \mu \mathrm{M}$ nobiletin and the corresponding concentration of $\mathrm{K} 252 \mathrm{a}$ with $(\bigcirc)$ or without $50 \mathrm{nM}$ NGF $(\bigcirc)$ and further incubated for $5 \mathrm{~h}$. The luciferase activity was relative to the control value. The assay was performed in quadruplicate. The value is expressed as an average. The bar represents S.D. *Significantly different from the value without NGF, $p<0.05$.

\section{Fig. 3}

The effect of K252a and PD98059 on the nobiletin-induced CRE-dependent transcription in the absence and presence of NGF. PC12 cells transiently transfected with a CRE reporter plasmid were preincubated with the indicated concentrations of K252a ( $\square)$, PD98059 ( $\square$ ), or K252a plus PD98059 $(\bigcirc)$ for 30min. Then, the medium 
was changed into fresh one that contained $10 \mu \mathrm{M}$ nobiletin and the indicated concentration of inhibitors in the absence (A) or presence of $50 \mathrm{nM}$ NGF (B) and further incubated for $5 \mathrm{~h}$. The luciferase activity was relative to the control value. The assay was performed in quadruplicate. The value is expressed as an average. The bar represents S.D. Significant differences among the three groups were evaluated using ANOVA. *Significantly different from the value with K252a, $p<0.01 . * *$ Significantly different from the value with K252a or PD98059 alone, $p<0.01$.

\section{Fig. 4}

The effect of nobiletin and NGF on Erk phosphorylation. (A) Nobiletin and NGF cooperatively increased Erk phosphorylation. PC 12 cells were incubated with $30 \mu \mathrm{M}$ nobiletin, $10 \mathrm{nM} \mathrm{NGF}$, or both for the indicated times. The cell lysates were recovered and processed for SDS-PAGE. The proteins transferred to the PVDF membrane were used for western blot with anti-phospho Erk antibody (upper panel) and anti-Erk antibody (lower panel). (B) The effect of inhibitors on the Erk phosphorylation induced by nobiletin and NGF. PC 12 cells were preincubated with $100 \mathrm{nM} \mathrm{K252a,} 50 \mu \mathrm{M}$ 
PD98059, or both for $30 \mathrm{~min}$. Then, the cells were incubated with $10 \mu \mathrm{M}$ nobiletin or nobiletin plus $50 \mathrm{nM}$ NGF in the presence of the inhibitors for $15 \mathrm{~min}$. The cell lysates were recovered for western blot as described above.

\section{Fig. 5}

Effect of the retrospective addition of NGF on the nobiletin-induced CRE-dependent transcription. PC12 cells were transiently transfected with a CRE reporter plasmid. The CRE-dependent transcription was measured by incubating with $10 \mu \mathrm{M}$ nobiletin for 5 hrs. NGF at $50 \mathrm{nM}(\bigcirc)$ was retrospectively added to the culture medium as indicated.

Control received the equal volume of PBS $(\mathbf{O})$. The assay was performed in quadruplicate. The value is expressed as an average. The bar represents S.D.

*Significantly different from the control value, $p<0.05$.

\section{Fig. 6}

The effect of NGF preincubation time on the nobiletin-induced CRE-dependent transcription. PC12 cells transiently transfected with a CRE reporter plasmid were 
preincubated with $50 \mathrm{nM}$ NGF from 0 to $120 \mathrm{~min}$ as indicated. Then, the medium was changed into fresh one that contained $10 \mu \mathrm{M}$ nobiletin with $(\bigcirc)$ or without $50 \mathrm{nM}$ NGF (O) and further incubated for $3 \mathrm{~h}$. The luciferase activity was relative to the control value. The assay was performed in quadruplicate. The value is expressed as an average. The bar represents S.D. *Significantly different from the value without preincubation, $p$ $<0.05$. * Significantly different from the value preincubated with NGF for 60 and 120 $\min , p<0.05$. 
Fig. 1
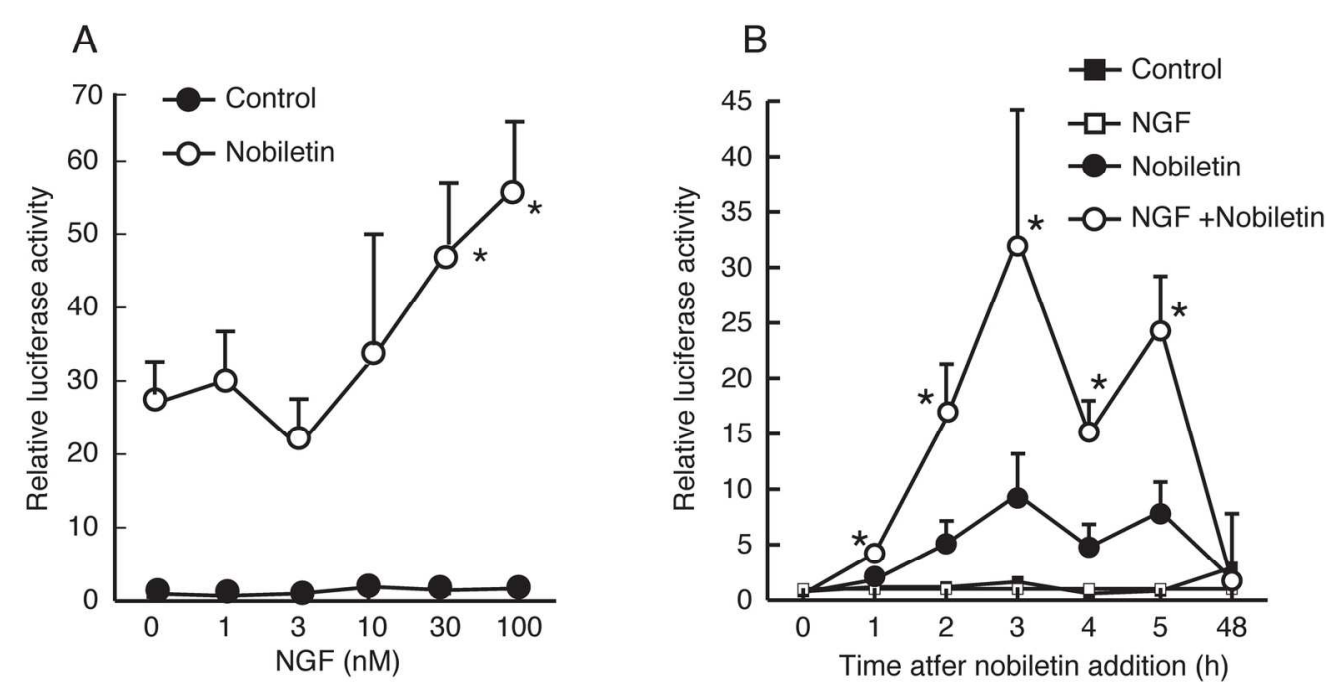

Fig. 1

$154 \times 91 \mathrm{~mm}(300 \times 300 \mathrm{DPI})$ 
Fig. 2

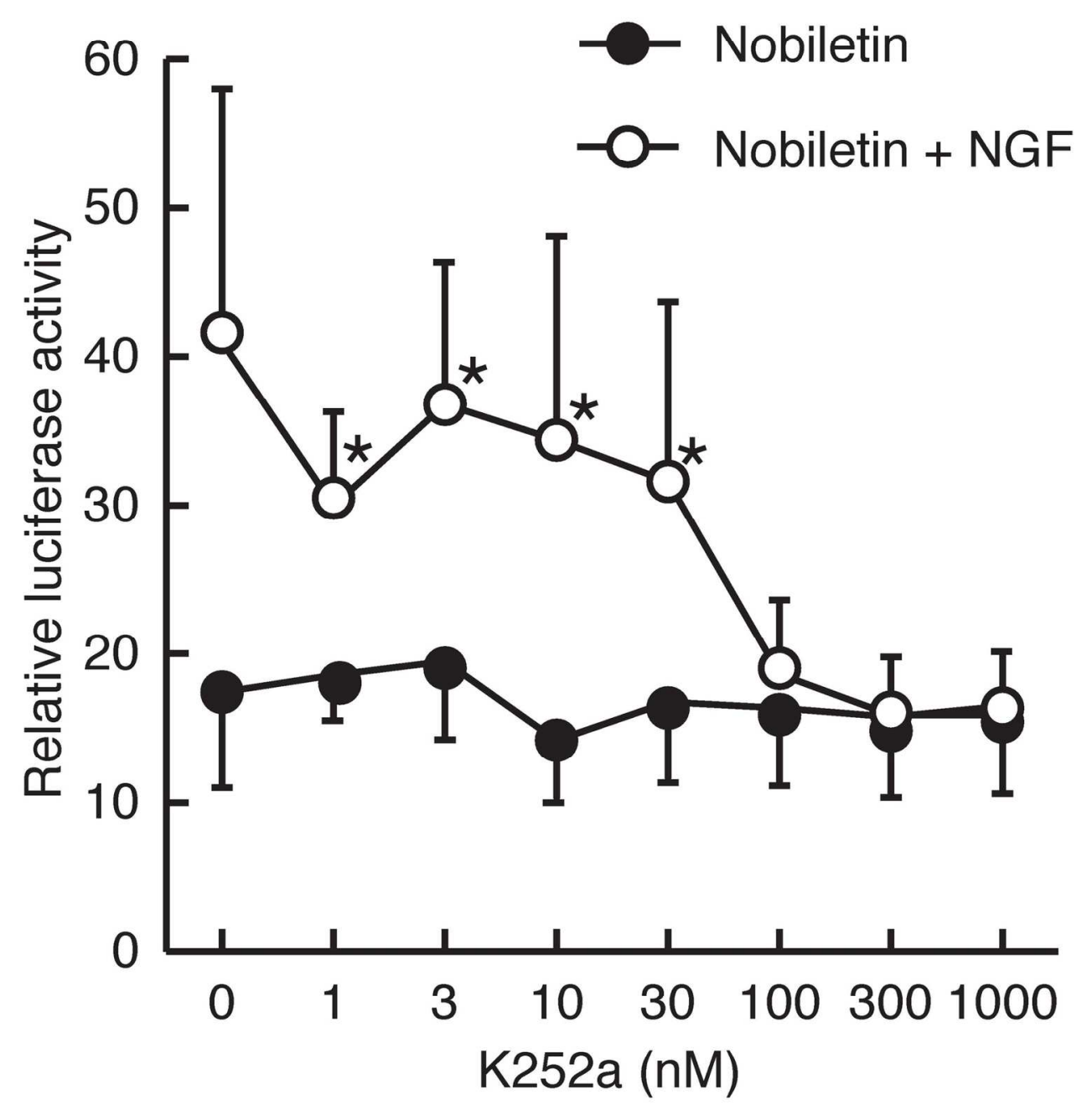

Fig.2

$146 \times 181 \mathrm{~mm}(300 \times 300 \mathrm{DPI})$ 
Fig. 3
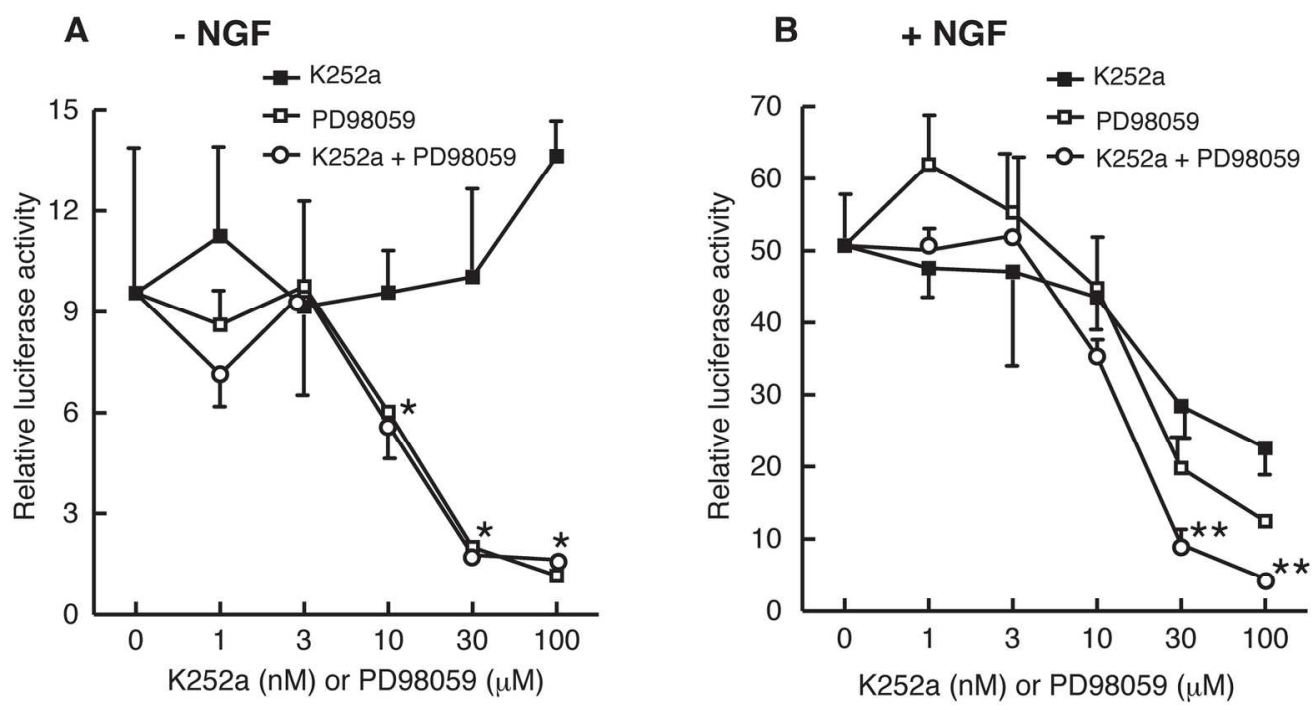

Fig. 3

$158 \times 97 \mathrm{~mm}(300 \times 300 \mathrm{DPI})$ 
Fig. 4

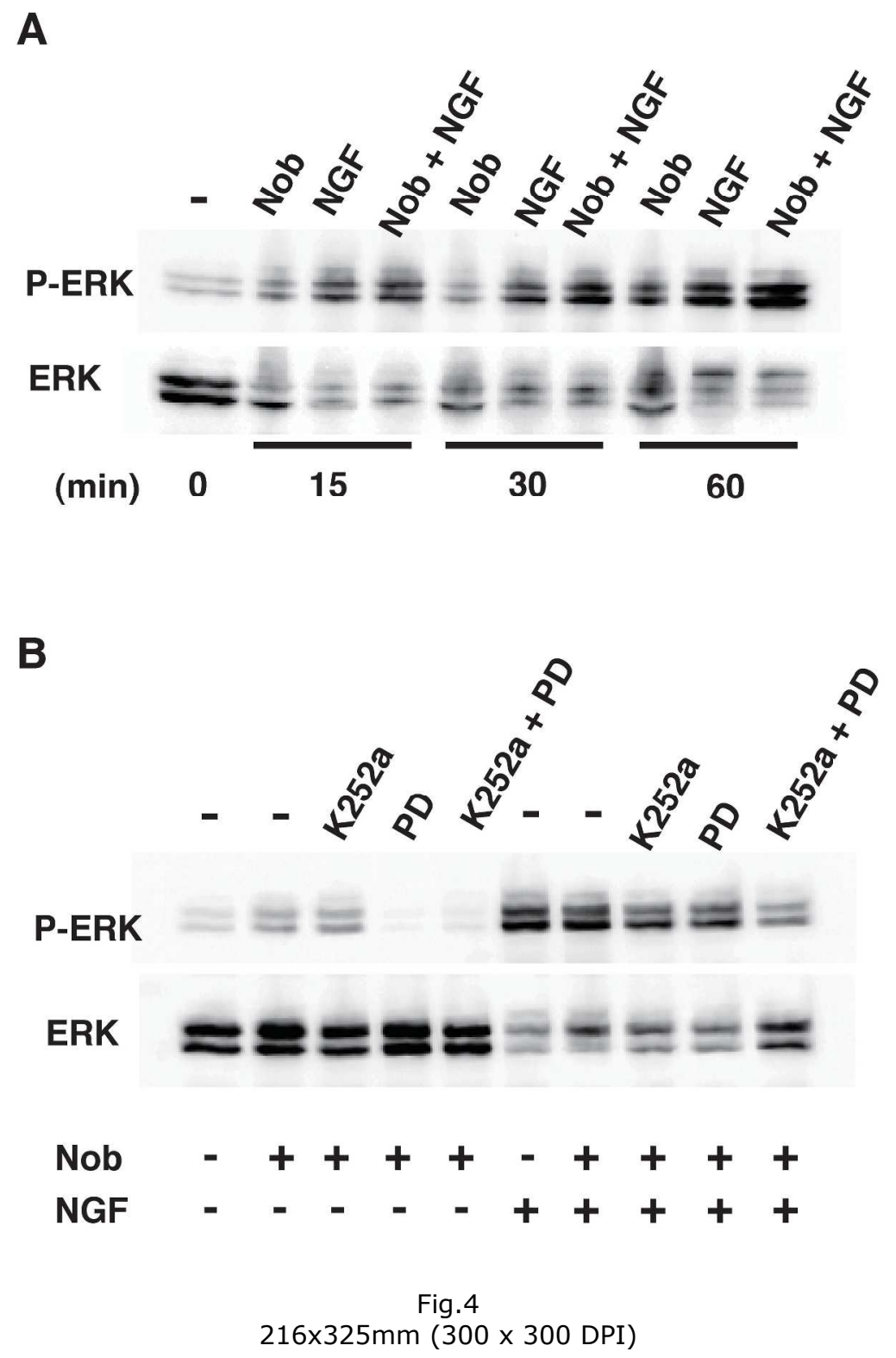


Fig. 5

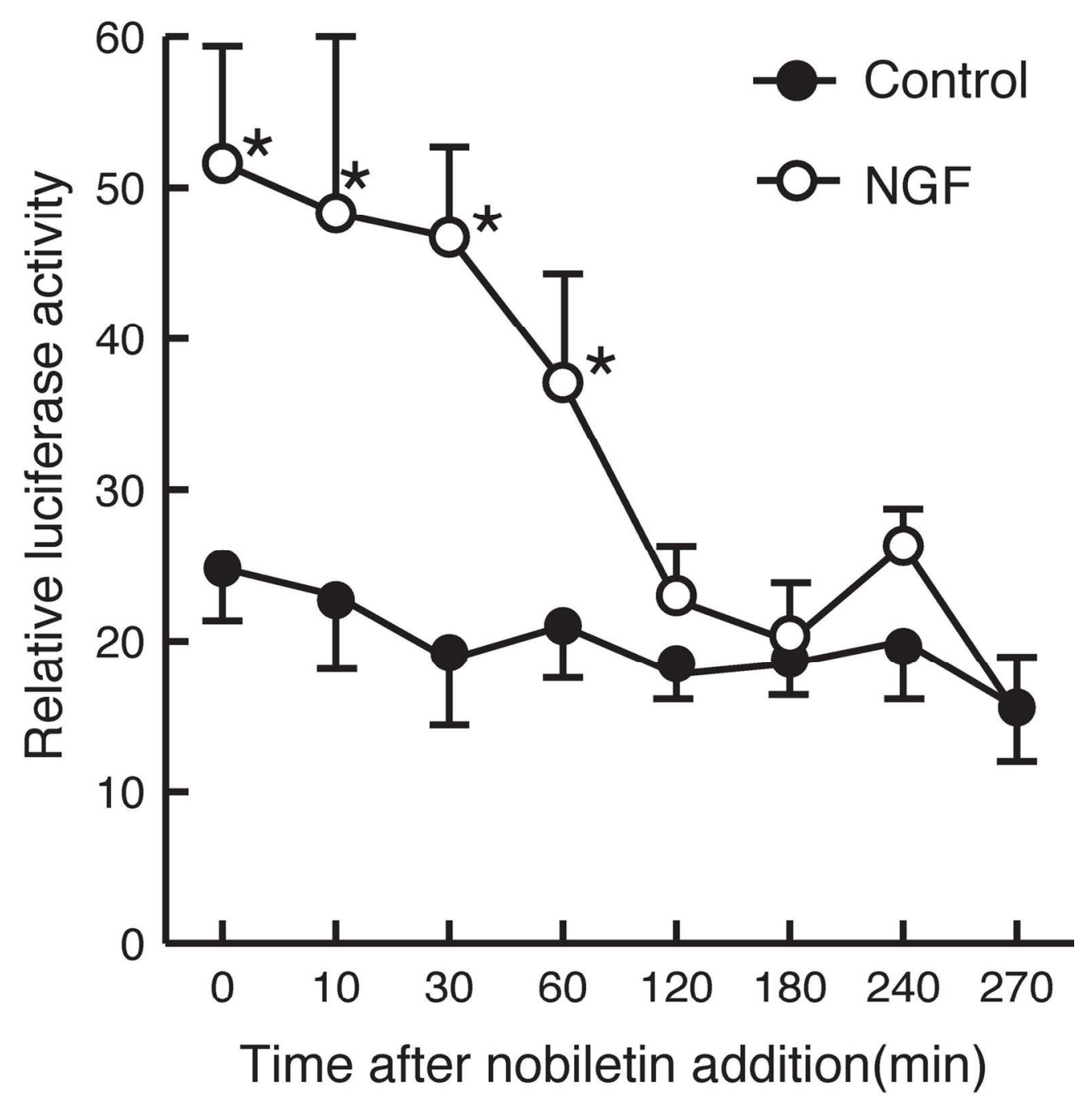

Fig. 5

$134 \times 156 \mathrm{~mm}(300 \times 300$ DPI $)$ 
Fig. 6

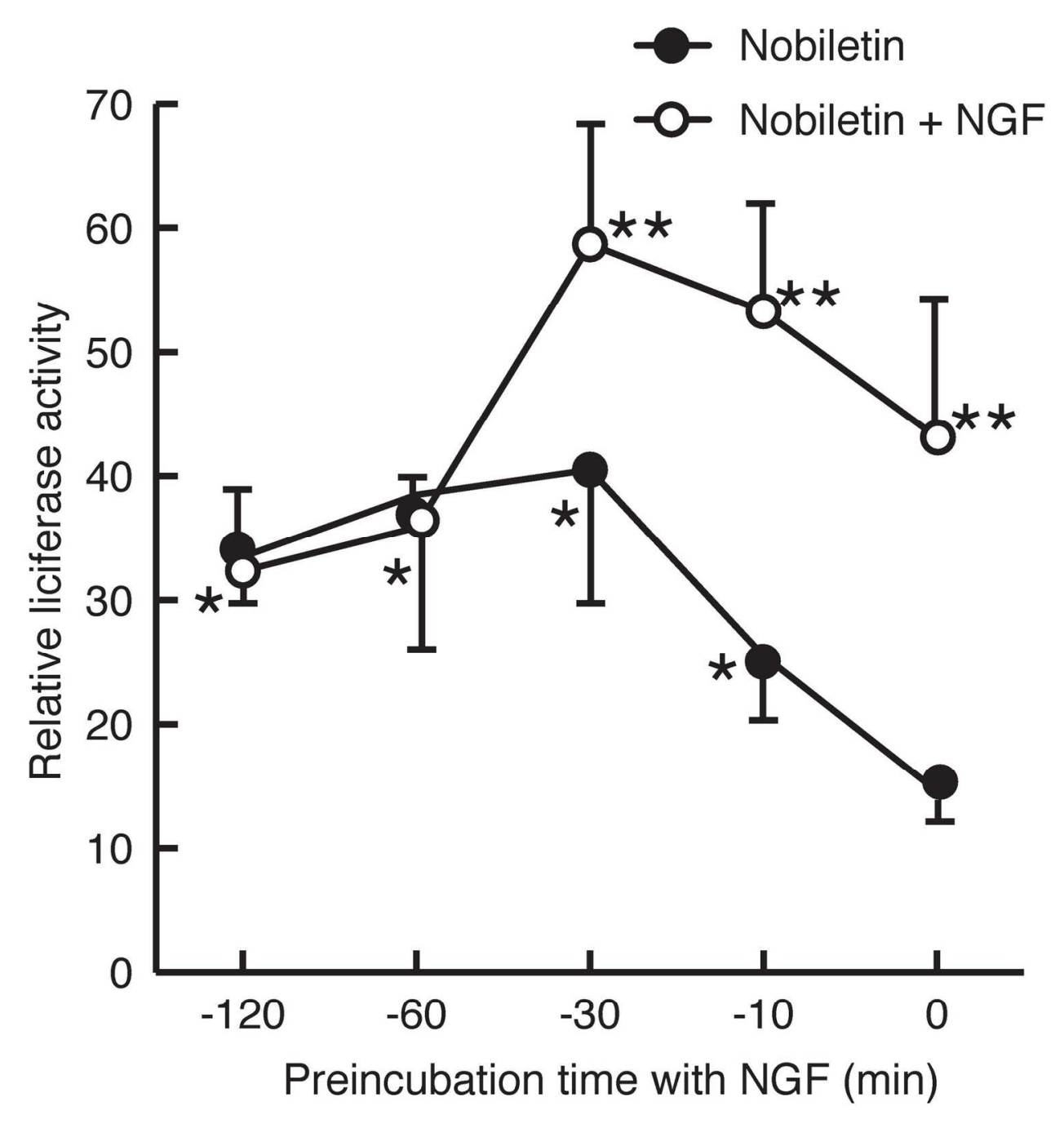

Fig. 6

$146 \times 182 \mathrm{~mm}(300 \times 300$ DPI $)$ 